\title{
TYPICAL ERRORS, ACCURACY CLASSES AND CURRENTLY EXPECTED ACCURACY OF INERTIAL MEASUREMENT UNITS
}

\author{
Attila VARGA* \\ PhD Student - Doctoral School of Applied Informatics and Applied Mathematics, Óbuda University, \\ Hungary, vargaa@stud.uni-obuda.hu \\ Tamas JANCSO \\ Associate Professor habil. Phd eng. - Alba Regia Technical Faculty, Óbuda University, Hungary, \\ jancso.tamas@amk.uni-obuda.hu

\section{Peter UDVARDY} \\ Associate Professor Phd eng. - Alba Regia Technical Faculty, Óbuda University, Hungary, \\ udvardy.peter@amk.uni-obuda.hu \\ *Corresponding author. E-mail: vargaa@stud.uni-obuda.hu
}

\begin{abstract}
Inertial measurement units can nowadays be found in a wide range of applications, from consumer electronics to precision surveying systems requiring high accuracy. IMUs are also widely used in aviation, especially for UAVs. The gyroscopes and accelerometers in IMUs have specific operating modes that would result in specific errors typical of such instruments. This paper reviews the typical errors of IMUs and the accuracy that can be achieved with these instruments today in each application area.
\end{abstract}

Keywords: inertial navigation; IMU; gyroscope; accelerometer; MEMS

\section{INTRODUCTION}

Inertial measurement units (IMUs) are nowadays one of the most commonly used navigation devices, alongside GNSS. Their development has come a long way from complex and heavy mechanical structures to devices based on microelectromechanical systems (MEMS) technology.

Although the sensors used in inertial measurement units, accelerometers and gyroscopes were known from earlier times, their combined use for positioning as inertial measurement units, only started during the Second World War. The main reason for this was the emergence of military equipment (e.g. ballistic missiles) whose guidance systems required inertial devices. Military needs were a strong driving force for the development of these devices. It was only in the 1970s that inertial navigation began to be used in civilian applications. [1] Today, inertial measurement units are used in a wide range of applications and are a common component in consumer electronics (e.g. mobile phones, computer games, etc.). Technology is advancing rapidly, and it is becoming cheaper and cheaper to obtain better, more accurate and more stable inertial systems.

\section{FUNCTIONS OF THE IMU}

An inertial measurement unit is a complex measurement system consisting of gyroscopes and accelerometers used to measure spatial displacement in an inertial frame (typically the Earth's inertial frame). In general, it is capable of measuring displacement in three directions in a straight line and rotation about three coordinate axes, interpreted in a spatially orthogonal coordinate system. Each measured value represents one degree of freedom. The inertial measurement system is often supplemented by other measuring instruments of physics quantity. Such instruments include a magnetic compass (magnetometer), barometric altimeter or temperature gauge. A measuring unit capable of measuring nine independent parameters ( 3 displacements, 3 rotations and 3 magnetic fields) is called a 9-degree-of-freedom system (or 9-DOF system). 
Inertial sensors alone can only measure relative quantities. The inertial measurement unit may be used as a stand-alone positioning system or may be used as a supplementary system to some other navigation system. It is often used as a complement to absolute positioning systems such as GNSS. High operating frequency of inertial measurement units can be used to increase the operating frequency of low operating frequency $(4-100 \mathrm{~Hz})$ systems like GNSS. Moreover, the navigation system remains operational even in the event of signal loss. For this reason, inertial measurement systems are nowadays increasingly integrated with GNSS systems. This results in an inertial navigation system (INS). This is a data fusion between GNSS and IMU and is itself capable of absolute position and orientation measurement. [2]

In integrated systems, a high level of sensor data fusion is achieved, minimising the errors of individual sensors. Today, inertial systems are available at low cost that can be used efficiently and easily without the need for deep mathematical knowledge (e.g. the Kalman filter) and provide measurement data with the accuracy required.

A typical inertial measurement unit used in aviation today consists of the following units:

- three accelerometers $(\mathrm{x}, \mathrm{y}, \mathrm{z})$

- three gyroscopes $(\mathrm{x}, \mathrm{y}, \mathrm{z})$

- $\quad$ three magnetometers $(\mathrm{x}, \mathrm{y}, \mathrm{z})$

- thermal sensor

- barometric altimeter

- digital signal processing unit (typically as embedded systems)

- power supply and control unit

- communication unit (standard interface)

In modern inertial measurement units, the analogue signals from the sensor are converted into digital signals, which are processed by a central logic unit (in practice an embedded system), which takes care of data fusion and error correction for each sensor. The processing unit is connected to the other systems of the receiving device via a digital interface.

\section{TECHNOLOGICAL BACKGROUND}

In the 1970s, the so-called strap-down systems appeared, where there was no longer a rotor mechanism. The first such systems used a ring laser gyroscope (RLG), which became really useful and widespread in the 1980s. It was also at this time that the fiber-optic laser gyroscope (FOG) appeared. Laser gyroscopes are used in places requiring high precision, such as aerospace, aeronautics, rocketry and various military applications. Their prices are extremely high, devices costing thousands or tens of thousands of dollars. For this reason, their use in industrial applications, including industrial UAVs, is rare.

Since the 1980s, microelectromechanical (MEMS) technology has developed rapidly, combining integrated circuit elements with mechanical components. Thanks to these inexpensive to produce MEMS sensors, inertial measurement units have now become widespread and widely used sensors in both gyroscopes and accelerometers.

Vibrating element MEMS gyroscopes (also called Coriolis Vibrating Gyroscopes, CVG) are the most widely used gyroscopes today, used almost exclusively for industrial systems and consumer electronics. These devices work by exploiting the Coriolis force phenomenon.[3]

A gyroscope made with MEMS technology is less accurate than a laser gyroscope, but has several advantages that make this type of gyroscope widely used:

- it is cheap to manufacture and the technology is mature,

- can be produced in a small size,

- low weight,

- low power consumption,

- fast initialisation,

- reliable, maintenance-free operation,

- resistance to environmental influences. 


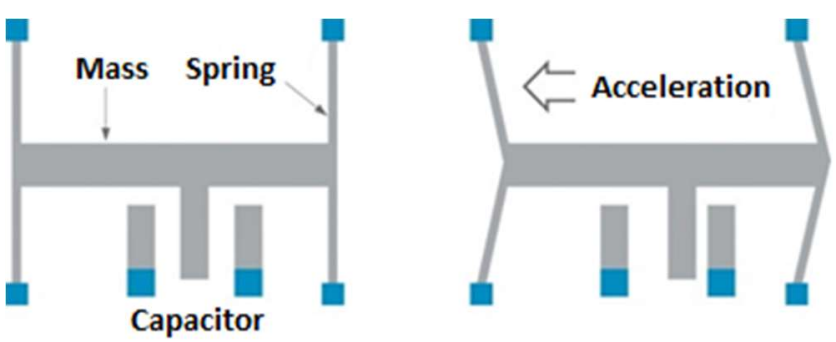

Figure 1. Schematic layout of MEMS based capacitive accelerometer

\section{TYPICAL IMU ERRORS}

Inertial measurement units can only measure relative displacement. An accelerometer measures relative linear displacement, while a gyroscope measures relative rotation. Therefore, the method is suitable for absolute positioning if the starting point of the displacement (or rotation) is known, or the initial velocity in the case of velocity.

The operation of a gyroscope is slightly different. There we determine angular rotation. The physical characteristic that can be measured is angular velocity. In the gyroscope, a signal is produced proportional to the angular velocity, the speed of rotation. By integrating the angular velocity once over time, we obtain the angular rotation. The integration operation already predicts that errors in the output will accumulate.

As in the any real measuring devices, the data provided by an inertial measurement unit will contain errors. However, the nature of these errors is quite specific compared to other instruments, due to the operational nature of inertial sensors. The measured signals are generated from the physically measured quantities (acceleration for displacement, angular velocity for rotation) by time integration. This means that the errors are also aggregated, integrated and increase continuously over time. When using inertial measurement units, one must be aware of these errors to use them correctly.

The gyroscope error is critical for the accuracy of the IMU. Due to geometric constraints, even very small errors in direction can lead to large errors in distance. The errors of gyroscopes and accelerometers are very similar in nature, but while for the gyroscope they are characterized by quantities related to rotation, for the accelerometer they are characterized by units related to linear motion.

The most common error in gyroscopes is drift, which is produced by integrating the errors in the measurement signal. Due to integration over time, this error increases continuously over time. Drift consists of two components: a constant bias and noise. These are typical errors in MEMS gyroscopes [4].

The bias is due to the fact that the gyroscope's sensor also provides an electrical signal at rest. This is continuously added to the output signal due to integration by time. This can be handled by measuring the constant error at the output of the gyroscope at rest for an extended period of time and then continuously subtracting this error during the subsequent measurement. The reason for the constant bias is to be found in the microelectromechanical design of the gyroscope. The vibrating element is moving in two planes at the same time. On the one hand, in the plane of vibration and, on the other hand, perpendicular to this plane, due to the Coriolis force. However, the deforming elastic element does not return to its original position even at rest due to internal friction and suffers some residual deformation. This deformation generates a continuous signal on the sensor. The displacement of the gyroscope is given by the angular increment that occurs over a unit of time (typically hours). This value is one of the most important parameters of an inertial measurement unit and is an indication of the accuracy and stability of the instrument. For example, the displacement of an industrial grade inertial unit is typically around $5 \%$ h.

The output of the MEMS gyroscope also shows a high frequency noise. This is due to the thermal behavior of the mechanical system, the failure of the electronic transducer and environmental effects (e.g. vibration). When this noise is integrated, some random value is resulted, which evolves irregularly over time (it can increase and decrease). This is called an angle random walk (ARW). It can be reduced by filtering high frequency noise. Its measurement is described by the noise density, measured in $\% / \sqrt{H z}$. 


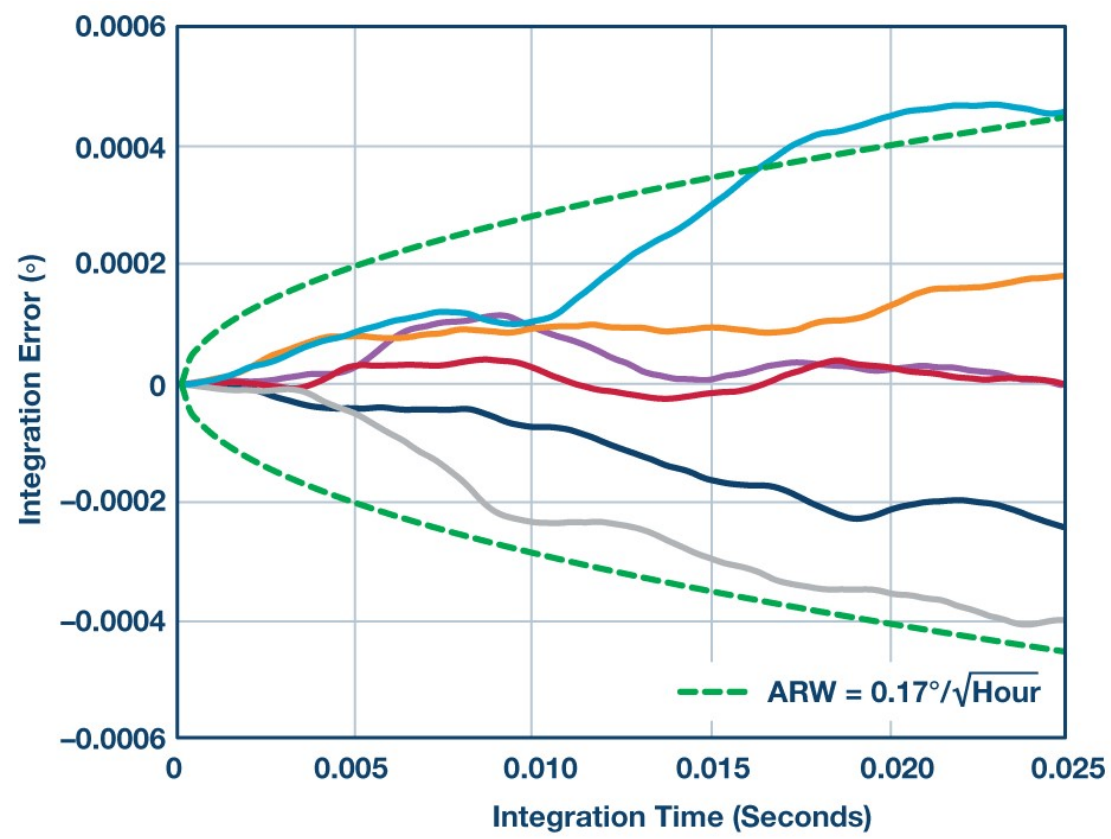

Figure 2. Time evolution of random angular displacement due to noise at the output of a gyroscope at rest and interpretation of ARW

Source: Mark Looney: Designing for Low Noise Feedback Control with MEMS Gyroscopes, AnalogDialogue, 2016/05 - URL: https://www.analog.com/en/analog-dialogue/articles/low-noise-feedback-control.html\#

Accelerometers built into the inertial unit are also used to compensate for the constant bias. The drift of gyroscopes measuring rotation about the $\mathrm{X}$ and $\mathrm{Y}$ axes can be compensated by accelerometers measuring gravity. The drift of rotation about the $\mathrm{Z}$ axis cannot be compensated in this way but can be compensated by a magnetometer measuring the earth's magnetic field.

The bias and drift of the accelerometer can be handled in a completely analogous way to the drift of the gyroscope. Given that the accelerometer measures the characteristics of linear motion, the drift is measured in $\frac{m}{s^{2}}$ and is often expressed in $\mathrm{mg}$ or $\mathrm{g}$, related to the acceleration of gravity. The unit of noise for accelerometers is $\frac{\mu g}{\sqrt{H z}}$.

In addition to the constant drift, manufacturers also specify the characteristics of the drift reproducibility. This is the drift repeatability, or bias repeatability, or turn-on to turn-on bias. Each time the sensor is turned on, the bias has a different value. This is because the physical state is always different when the system is initialised, and therefore the constant error is also different. The parameter therefore gives the typical amount of drift error between two power-ups, in $\%$ h for the gyroscope and $\frac{m}{s^{2}}$ for the accelerometer. The smaller the drift repetition error, the smaller the uncertainty in the constant error, so the more efficiently this error can be compensated for (simply subtract from the signal provided) after initialisation.

Manufacturers usually also specify the drift during operation, which indicates the drift from the rest position. The unit of measurement is $\%$ h for gyroscopes and $\frac{m}{s^{2}}$ for accelerometers.

Drift is also present in the laser gyroscope, but it is one or two orders of magnitude smaller, depending on the accuracy class. The laser gyroscope has no mechanical parts, so drift due to material properties does not occur.

However, the optical path of the laser beam can vary due to different external conditions. For example, the optical path can change due to temperature changes, although designers try to achieve designs that are less sensitive to temperature effects. Noise from electronic signal processing also stresses the signals from the laser gyroscope, so drift that increases with time will also be reflected in the laser sensors. The rate is $1-0.1^{\circ} / \mathrm{hr}$ or even less.[5]

Another characteristic errors of inertial measurement unit are the so-called scale factor and the degree of linearity of the scale factor. 


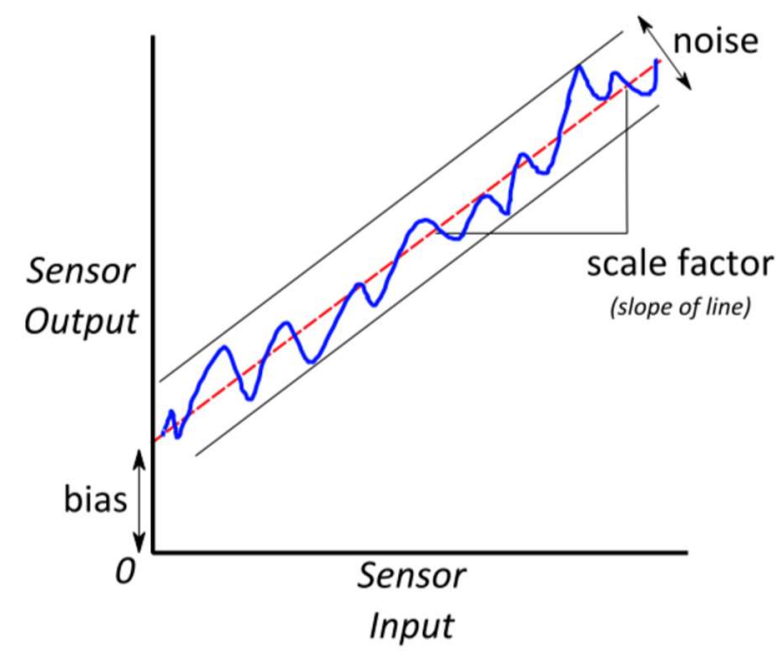

Figure 3. Illustration of some of the errors (scale factor, bias, noise) of inertial sensor.

Source: IMU Errors and Their Effects, NovAtel

https://hexagondownloads.blob.core.windows.net/public/Novatel/assets/Documents/Bulletins/APN064/APN064.p $d f$

The scale factor (see Figure 3.) gives the relationship between the input signal (e.g. displacement) and the sensor output signal (e.g. voltage). It is ideally 1 , in reality a slightly different number. It is effectively the slope of the graph of the relationship. Manufacturers give the percentage value of the magnification factor in parts per million (ppm).

Besides the scale factor, the scale factor linearity of the relationship between input and output is also an important parameter. Ideally, the graph of this function is a straight line. In the real sensor, however, this linearity is not achieved. It is also given as a percentage, in ppm.

Occasionally, the geometric errors of the sensors are also given for the inertial units, which are determined during the calibration after manufacture. This is also known as the perpendicularity or orthogonality error, which means that the sensor is installed with some error in the axis of the orthogonal coordinate system interpreted for the inertial system. This causes components in other directions to appear in the measurement result when measuring acceleration or rotation in a given direction. Manufacturers usually specify it in microradians.

\section{ACCURACY CLASSES}

Inertial measurement units are divided into categories, or accuracy classes, according to the reliability and stability required for each application. However, there is no clear system of categories, the categories and accuracy classes are highly manufacturer specific and the boundaries of the parameters of each class are not clear. Moreover, as technology evolves, these categories are constantly changing and the parameters for accuracy and stability are constantly improving. For example, the inertial measurement units found in today's consumer electronics products met the criteria required for industrial applications just a few years ago. However, despite the uncertainties, it is possible to distinguish a few categories, mainly for specific applications, for which the technical parameters and the technologies used can be clearly defined. A possible and widely used categorisation, which is also used by VectorNav, is: naval grade, tactical grade, industrial grade and user electronics or automotive grade. [6]

The best performing and most accurate inertial systems commercially available are those for marinenavigation applications where stable operation over long periods of time is a requirement. Even more accurate and stable than these are devices used in military weapons and space applications, but these are strategic products. The latter are sometimes treated as a separate category and referred to as strategic grade. This includes inertial measurement units used on civil and military aircraft. Instruments in this category have a horizontal error of $12 \mathrm{~mm}$ after 10 seconds, $0.44 \mathrm{~m}$ after 10 minutes and $1.6 \mathrm{~km}$ after one hour. The accuracy of very high quality IMUs used for navigation purposes, for example on 
warships and submarines, nowadays reaches a level where the horizontal drift does not exceed $1.8 \mathrm{~km}$ during a 24-hour autonomous operation. In all such instruments, some form of laser gyroscope is used. Their price can be in excess of $\$ 100,000$, and the most accurate marine instruments can cost up to a million dollars. The drift of a gyro is $0.01 \%$ or less.

The tactical grade is also primarily the domain of military applications. However, the tactical classification means that it is required to provide accurate position and attitude data in a shorter time frame. Here, a horizontal error of $155 \mathrm{~mm}$ in 10 seconds and $5.3 \mathrm{~m}$ in 10 minutes is allowed. These devices are designed for shorter operating times in devices such as guided weapons, antenna stabilisation platforms and unmanned aerial vehicles (UAVs). In terms of technological implementation, both laser devices and MEMS technology are common. Their price is around 1000 USD. Gyroscope drift is around $1-0.1^{\circ} / \mathrm{h}$.

The lowest segment of inertial measurement units is the costumer electronics grade. This is often referred to as the automotive grade, because one of the main users of this type of inertial unit is the automotive industry. These units are often not used for positioning, but act as motion sensors, so that significant cumulative errors are not a problem during a few minutes of operation. Typical applications in the automotive industry include automatic drive train control, airbag actuation, or motion sensors for various electronic toys and mobile phones. The drift of such gyroscopes can be $10-100^{\circ} / \mathrm{h}$.

Between the automotive level and the tactical level lies the category of industrial grade devices. They are invariably based on MEMS technology, i.e. for industrial applications (UAVs, geodetic surveying, mobile mapping, photogrammetry, etc.) they are in an affordable price range and have a reasonable drift. Their adequate accuracy for inertial navigation is ensured by a processing unit with a suitable signal filter and good calibration. Their drift is typically $3-5^{\circ} / \mathrm{h}$.

\section{CALIBRATION}

Calibration is crucial for the accuracy and stability of inertial measurement units. Calibration is an essential procedure for inertial measurement units of a higher accuracy class (navigational and tactical), which are essentially custom-made. In the manufacturing process, MEMS inertial measurement units for industrial use and inertial measurement units for user electronics devices are manufactured using the same technology. The difference in the end result is the calibration. This compensates for some of the errors, in particular those due to permanent misalignment.

Inexpensive inertial measurement units of the consumer electronics accuracy class do not require calibration, they are based on the nominal parameters used at the time of design. For the industrial accuracy class, the inertial measurement unit is individually calibrated during manufacture and the resulting parameters are burned into the processing unit. The drift of the same microelectromechanical device is about an order of magnitude less if the device is individually calibrated.

For inertial measurement units, in addition to vibration, the operating temperature causes significant errors. In MEMS devices, the elasticity of the mechanical elements varies, because the elastic modulus of an elastic element is temperature dependent. Silicon, the material most commonly used for micromechanical devices, has elastic properties very similar to metals, especially steel. Unlike metals, however, it does not yield. The modulus of elasticity of monocrystalline silicon in range of 130 and $185 \mathrm{GPa}$. The modulus of elasticity is dependent on direction, so that a range is given for this value. It decreases with increasing temperature. Thus, changing temperature will have a different output for the same input (force) in MEMS sensors, and it is not a linear relationship.[7]

For calibrated devices, calibration is usually performed at room temperature (around $20-25^{\circ} \mathrm{C}$ ) and a given accuracy is guaranteed within a given temperature range (e.g. $\left.\pm 5-10^{\circ}\right)$. Many manufacturers also perform calibration over a wider temperature range (e.g. -40 to $80^{\circ} \mathrm{C}$ ) and then burn the resulting parameters into the signal processing unit of the inertial measurement unit. Such calibration can achieve tactical level accuracy with MEMS-based inertial measurement units. 


\section{DEVELOPMENT TRENDS}

The trend in inertial measurement units in recent years has been a steady reduction in geometric dimensions, weight and price, with an increase in accuracy and stability.

This is particularly true for MEMS technology sensors. MEMS sensors are cheap and mass-produced products. Its main raw material, crystalline silicon, is cheap and abundantly available. In addition to silicon, its compound, quartz single crystal, consisting of silicon dioxide, is increasingly used for inertial sensors.

In recent years, the accuracy of inertial devices has steadily increased. A significant increase in accuracy is no longer expected from MEMS sensors, as the mechanical elements in MEMS sensors mean that the accuracy can no longer be increased significantly. For this technology, further increases in accuracy can be achieved mainly through sensor fusion and improvements in signal processing.

The price of inertial measurement units using laser gyroscopes is also decreasing. However, size reduction is not a simple solution for laser instruments. The longer the optical path, the more accurate the laser gyroscope. This requires a larger mirror system for a ring gyroscope and a longer fiber-optic wire for a fiber-optic instrument.

There is also a market trend towards the integration of inertial measurement and GNSS instrumentation into a single unit, i.e. manufacturers are increasingly offering inertial navigation systems. These devices provide a solution for the fusion of inertial unit and GNSS (in most cases RTK GNSS) data within a single system, providing homogeneous position and attitude (orientation) data.

Professional devices for industrial use contain embedded systems that take care of the signal processing. To this end, the device is provided with a development environment and, in most cases, a graphical user interface (GUI).

It is now common practice in industrial systems to incorporate a Kalman filter in the signal processing units to fuse data from different sensors (accelerometers, gyroscopes, magnetometers, or GNSS in integrated devices). This allows signals with different sampling frequencies and different types of error to be processed together, thus minimising errors. In essence, the use of a Kalman filter is the means to ensure that industrial or tactical level of accuracy can be achieved using sensors based on MEMS technology. (The Kalman filter gives the best estimate of the new state of system based on the past and current state of the system, usually by means of error minimisation based on the method of least squares.)

\section{References}

[1] A. D. King: Inertial Navigation - Fourty Years of Evolution, in: GEC Review, 1998. Vol. 13. No. 3. - URL: https://www.imar-navigation.de/downloads/papers/inertial_navigation_introduction.pdf [2] A. Chatfield: Fundamentals of Hight Accuracy Inertial Navigation, Volume 174, Progress in Astronautics and Aeronautics, American Institute of Aeronautics and Astronautics, Reston, Virginia, 1997, ISBN 1-56347-243-0, pp. 13-15.

[3] Cenk Acar, Andrei Shkel, MEMS Vibratory Gyroscopes - Structural Approaches to Improve Robustness, Springer, 2009, ISBN 978-0-387-0953-6, pp. 8-9.

[4] IMU Errors and their Effects, NovAtel - URL: https://hexagondownloads. blob.core.windows.net/public/Novatel/assets/Documents/Bulletins/APN064/APN064.pdf

[5] Hervé C. Lefèvre: The Fiber-Optic Gyroscope, Artech House, 2014, ISBN 978-1-60807-695-6, pp. $45-47$.

[6] What is an Inertial Measurement Unit? VectorNav, Intertial Navigation Articles https:/www.vectornav.com/resources/inertial-navigation-articles/what-is-an-inertial-measurementunit-imu

[7] Paul L. Bergstorm, Gary G. Li: Inertial Sensors, in: The MEMS Handbook (editor: Mohamed Gad-el-Hak), CRC Press, 2002, ISBN 0-8493-0077-0

Received 06, 2021, accepted 07, 2021

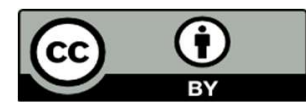

Article is licensed under a Creative Commons Attribution 4.0 International License 